\title{
A preparation and Characterization of Functionally Graded Aluminum Alloy Based Composite Via Casting Route
}

\author{
Mohammed M. Abdullah \\ Mechanical Engineering Department \\ College of Engineering, Mosul University, Mosul - IRAQ
}

\begin{abstract}
In the current study, stir and centrifugal casting apparatus have been designed and constructed for the preparation of cast functionally graded aluminium alloy (AA4043) based composites containing Silicon Carbide (SiC) reinforcement particles. Hardness of different cast functionally graded composites (FGCs.) processed with different rotating speeds have been investigated. Unreinforced base alloy and cast gravity composite corresponding to the composition of matrix alloy of cast FGCs have also been cast and characterized for the purpose of comparison. The present study aims to understand the influence of rotating speed of mould on the reinforcing particles distribution and hardness of the resulting cast FGCs. It was found that increasing the processing speed of mould increases the level of distribution of reinforcing particles from the inner to the outer region. At a given processing speed, hardness increases significantly with increasing the radial distance for the both top and bottom surfaces and could be attributed to the segregation of the reinforcing particles at the outer region.
\end{abstract}

Key Words: Functionally graded composite: Processing speed: SiC particles.

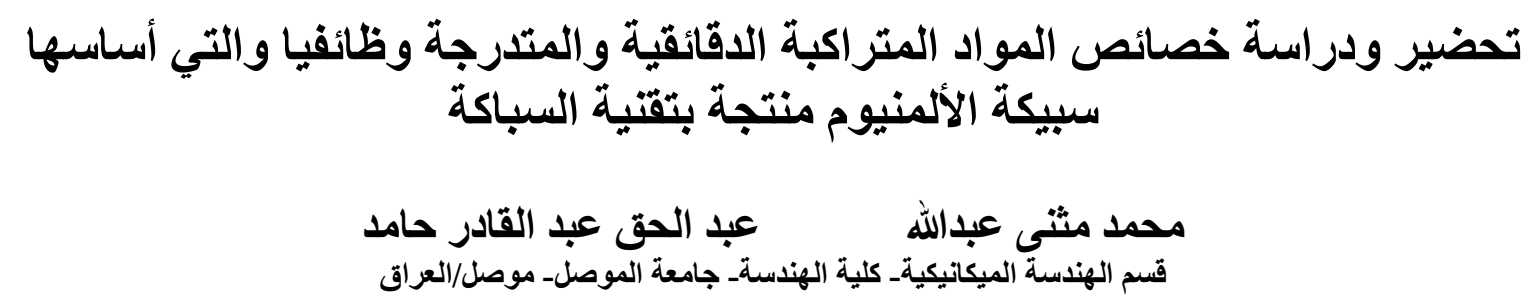

\section{الخلاصة}

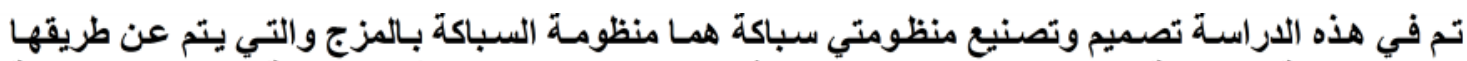

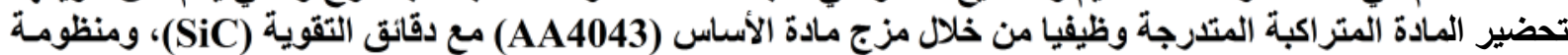

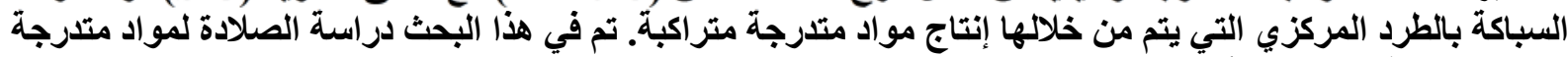

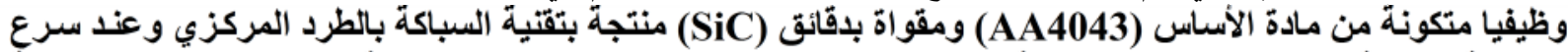

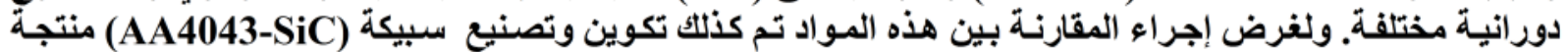

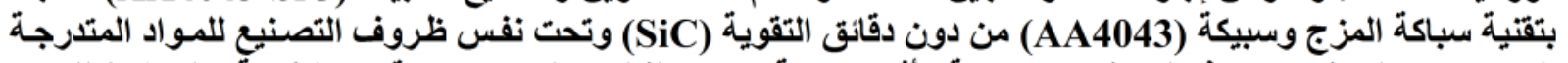

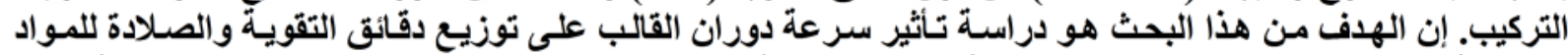

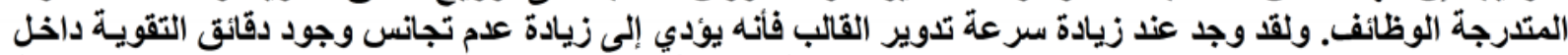

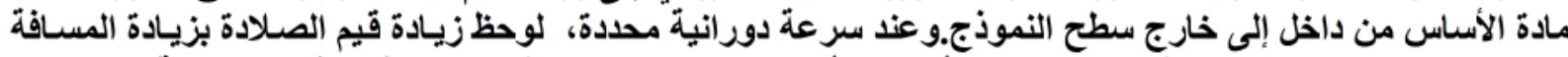

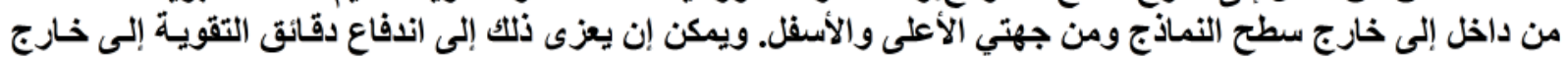

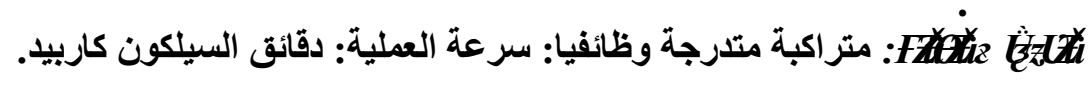




\section{1- Introduction:}

Composite materials are produced when two different materials are mixed to give a combination of properties that cannot be attained in original materials. Composites may be selected to give unusual combinations of stiffness, strength, weight, high temperature performance, corrosion resistance, hardness and conductivity [1,2]. Functionally Graded Materials (FGMs) are motivated by the need for properties that are unavailable in any single material and the need for graded properties to offset adverse effects of discontinuities for layered materials. Thus, in FGMs, the constituents vary in some direction enabling these materials to provide unique performance [3].

The best method to fabricate of the FGMs is the centrifugal method. The technique uses the centrifugal force generated by a rotating cylindrical mould to throw the molten metal against the mould wall and forms the desired shape. In centrifugal casting the mould rotates at a predetermined speed [4]. A controlled non-uniform microstructure with continuously changing properties FGMs reinforced with different ceramic particle could be produced by centrifugal casting technique to obtain these graded properties, which are not achievable in monolithic or homogenous materials [5]. Zhang et.al. [6] have produced the FGMs by in-situ centrifugal casting that used $\left(\mathrm{Mg}_{2} \mathrm{Si}\right)$ as a reinforcement particles and $\mathrm{Al}$ as a matrix material. The mould is shaped as tube. The results show the outer region of tube having more $\mathrm{Mg}_{2} \mathrm{Si}$ than the inner region of tube. Yoshimi et al [7] have studied the microstructures and composition gradients in Al-SiC, Al-Shirasu (volcanic eruptions commonly found in south Kyushu in Japan), $\mathrm{Al}-\mathrm{Al}_{3} \mathrm{Ti}, \mathrm{Al}-\mathrm{Al}_{3} \mathrm{Ni}$ and $\mathrm{Al}-\mathrm{Al}_{2} \mathrm{Cu}$ FGMs have been investigated. The Al$\mathrm{SiC}$, Al-Shirasu and $\mathrm{Al}-\mathrm{Al}_{3} \mathrm{Ti}$ FGMs are fabricated by the centrifugal solid particle method where the distribution particles of $\mathrm{SiC}$, Shirasu and $\mathrm{Al}_{3} \mathrm{Ti}$ are solids in the melts. The results show that the volume fraction of the $\mathrm{SiC}$ and $\mathrm{Al}_{3} \mathrm{Ti}$ increases towards to the outer region due to increasing the $\mathrm{G}$-factor. On the other hand, $\mathrm{Al}-\mathrm{Al}_{3} \mathrm{Ni}$ and $\mathrm{Al}-\mathrm{Al}_{2} \mathrm{Cu} \mathrm{FGMs}$ are fabricated by the centrifugal in-situ method where $\mathrm{Al}-\mathrm{Al}_{3} \mathrm{Ni}$ and $\mathrm{Al}-\mathrm{Al}_{2} \mathrm{Cu}$ systems have lower liquidus temperatures than the processing temperatures to compare these microstructures with ex-situ composites. Zenon [8] has fabricated and characterized the FGMs Al-Mg alloys with in-situ particles $\left(\mathrm{AlB}_{2}\right)$ composites using centrifugal casting. The results show that the in-situ $\mathrm{AlB}_{2}$ particles segregation toward the external zone of the cast piece, resulting in higher hardness and micro-hardness in this region.

The present research work have been directed to the influence of key processing parameters, namely processing mould speed on the microstructure has been determined and their impact on the hardness behaviour of the resulting FGCs has been evaluated. The knowledge base generated through this study is expected to lead to an understanding of the relationship between rotational speed, microstructure and hardness and may contribute to the identification of critical factors of processing in order to control the properties of these FGCs. Moreover, it is, also, expected to offer a better understanding of the potential and limitations of FGMs, which have already emerged as an advanced engineering material.

\section{2- Experimental Work:}

Commercial Aluminium - Silicon (AA4043) was chosen as the matrix alloy for studies on current cast FGCs. The molten Al-Si alloy was, also, alloyed by the addition of the required amount of Magnesium $(\mathrm{Mg})$ of $2 \mathrm{wt} . \%$. The presence of magnesium is well known to enhance the wettability of $\mathrm{SiC}$ particles in molten aluminium alloy due to a limited chemical 
reaction at the particle-alloy interface [2]. The chemical compositions of the commercial aluminium-silicon alloy, in total weight percent, as determined by optical spectrometer analyzer (portable-X-MET 3000 TX), used in solidification processing of different cast FGCs, composites and unreinforced alloys are given in Table (1). The chemical analysis was determined by the help of a workshop center at Northerner Cement Company (MosulNineveh).

Table (1): Chemical composition of the Aluminium Alloy (AA4043).

\begin{tabular}{|c|c|c|c|c|c|c|}
\hline $\begin{array}{c}\text { Aluminium } \\
\text { Association Grade }\end{array}$ & $\begin{array}{c}\mathrm{Cu} \\
\text { wt.\% }\end{array}$ & $\begin{array}{c}\mathrm{Ni} \\
\text { wt.\% }\end{array}$ & $\begin{array}{c}\mathrm{Si} \\
\text { wt.\% }\end{array}$ & $\begin{array}{c}\mathrm{Fe} \\
\text { wt.\% }\end{array}$ & $\begin{array}{c}\mathrm{Zn} \\
\text { wt.\% }\end{array}$ & $\begin{array}{c}\mathrm{Al} \\
\text { wt.\% }\end{array}$ \\
\hline AA4043 & 0.15 & 0.01 & $4.5-6$ & 0.59 & 0.12 & Balance \\
\hline
\end{tabular}

SiC powder with average particle size of $231 \mu \mathrm{m}$ was selected on the basis of reasonably low cost and easy availability, Also, the $\mathrm{SiC}$ particle, is well known, as a highly wear resistance and of good mechanical properties, including high-temperature strength and thermal shock resistance. The selected $\mathrm{SiC}$ particles were manufactured by Avonchem, Mseclesfied, Cheshire, United Kingdom (UK).

A batch type of stir casting set-up, as shown in Fig. 1 was used in this investigation, for the fabrication processing of all different cast FGCs, composites and unreinforced base alloy. The set-up consisted of a melting unit and a stirring arrangement. The melting unit was an electrical resistance heating vertical furnace. A calibrated chromel-alumel thermocouple was used to control the temperature of the furnace by placing it close to the wall. The temperature controller, having a range of $0-1400{ }^{\circ} \mathrm{C}$ with a control accuracy of $\pm 5{ }^{\circ} \mathrm{C}$, was used. For melting of aluminium alloy, a cylindrical tapered clay-graphite crucible with flat bottom was used. The crucible had an average diameter of $125 \mathrm{~mm}$ and was placed inside the furnace as shown in Fig.1. The stirrer was driven by 1/2 Horse Power (HP) motor having four rated speeds of 700, 900, 1100 and $1300 \mathrm{rpm}$ for stirring the melt. The motor of the stirrer was held rigidly over the furnace by a gripping arrangement centrally fixed with steel frame structure of the set-up as shown in Fig.1. The stirrer was fixed to the shaft of motor by gripping coupling arrangement. The rigid base structure of the stirrer arrangement was containing a lifting device for controlling the position of the stirrer in the crucible. A suitable funnel was placed near the stirrer rod for incorporating reinforcing particles into molten aluminium alloy. All composites and unreinforced base alloy were prepared by the above arrangement [9].

Figures 2 and 3 show the constructed centrifugal casting set-up used in the current study [9]. The centrifugal set-up consisted of a rotating steel mould as shown in Fig. 4, electrical motor, heating system and temperature controller. The steel mould was mounted on one end of the vertical shaft of the electrical motor by using coupling arrangement unit. The circular heating elements were kept around the mould, leaving sufficient clearance, in order to heat the mould and delay the solidification process of the composite slurry. The electrical connection to the heating element was driven through an autotransformer to control the power input to the heaters. A calibrated chromel-alumel thermocouple was used to control the temperature of the chamber by placing it close to the mould wall. The following subdivision paragraphs explain and describe in more details all the essential parts included in the above centrifugal set-up. 
No. 1

FEB. 2015

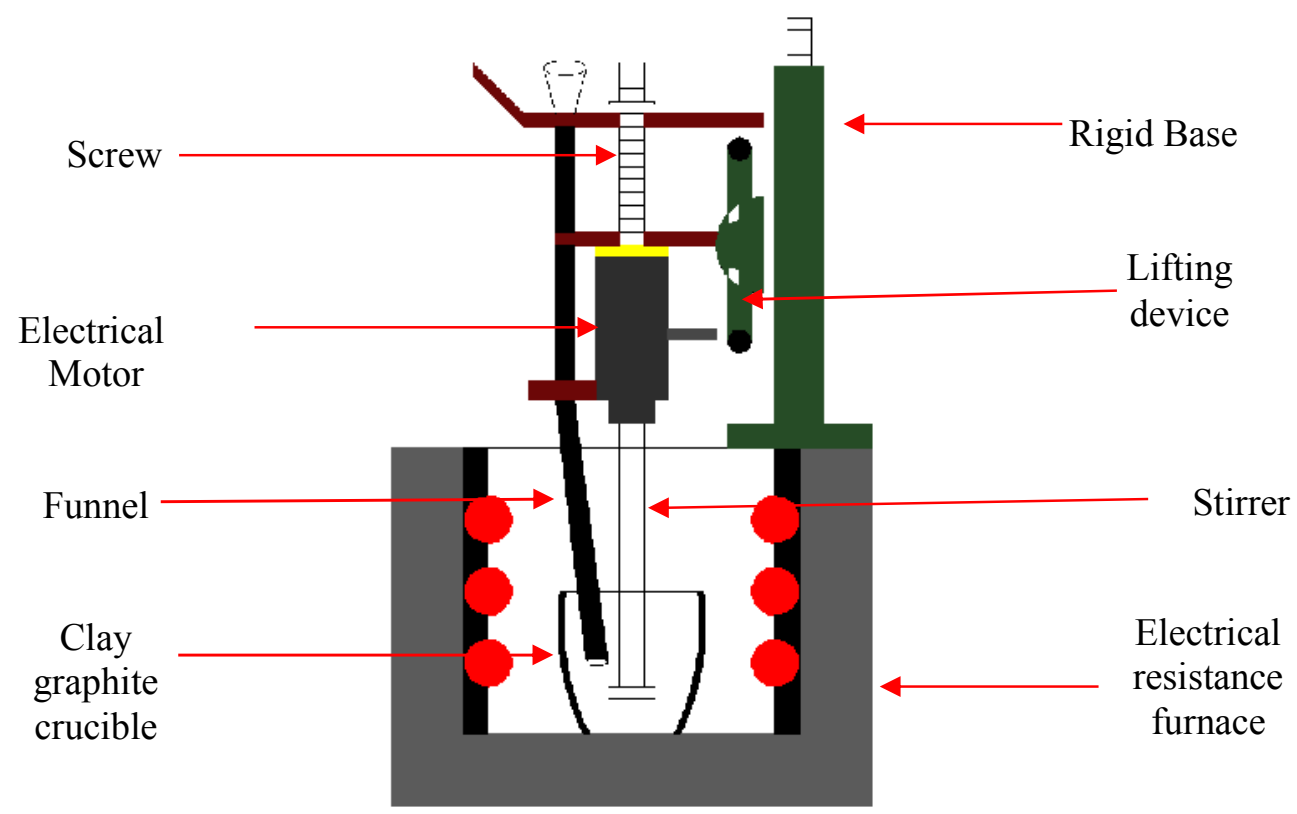

Figure (1): Diagram showing the stir casting arrangement used in the current

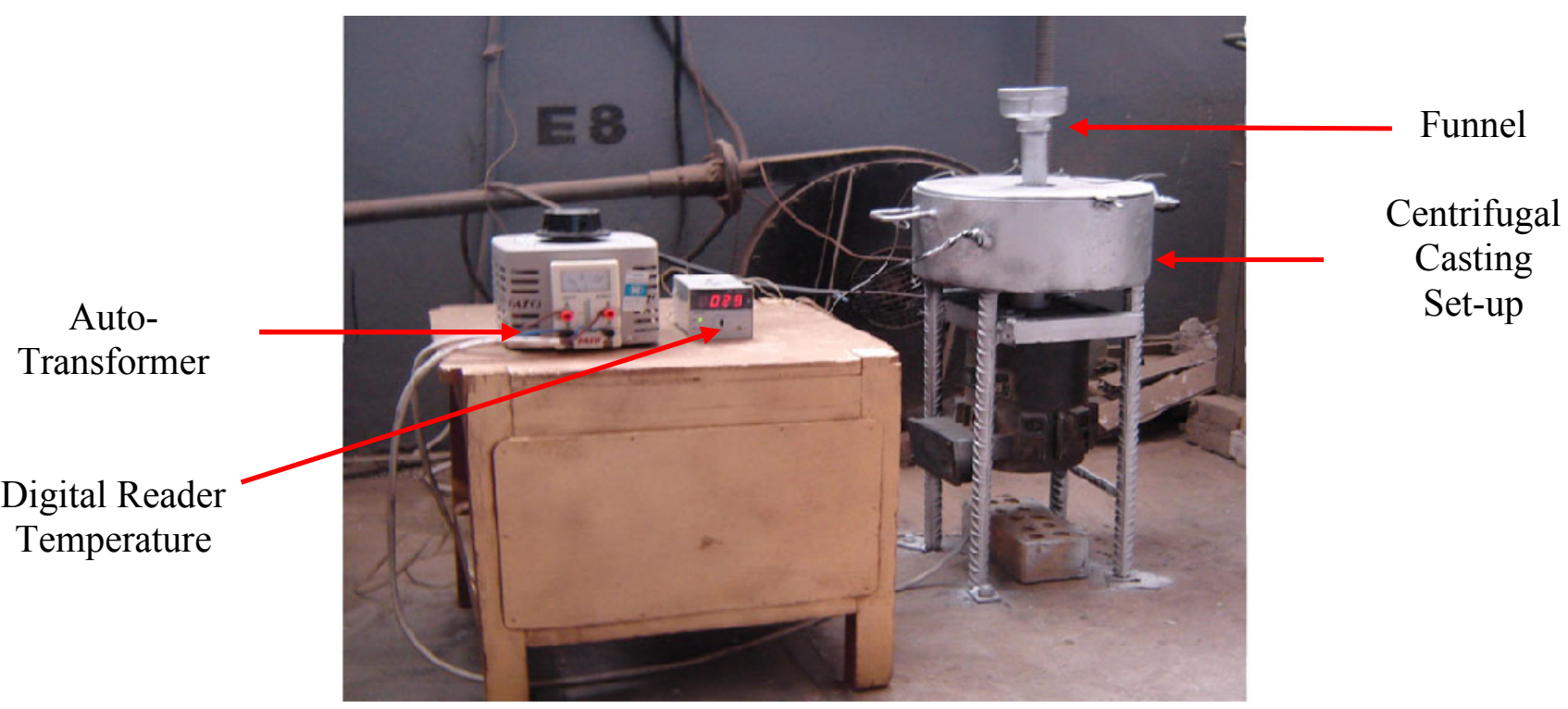

Figure (2): Photograph of centrifugal casting apparatus. 


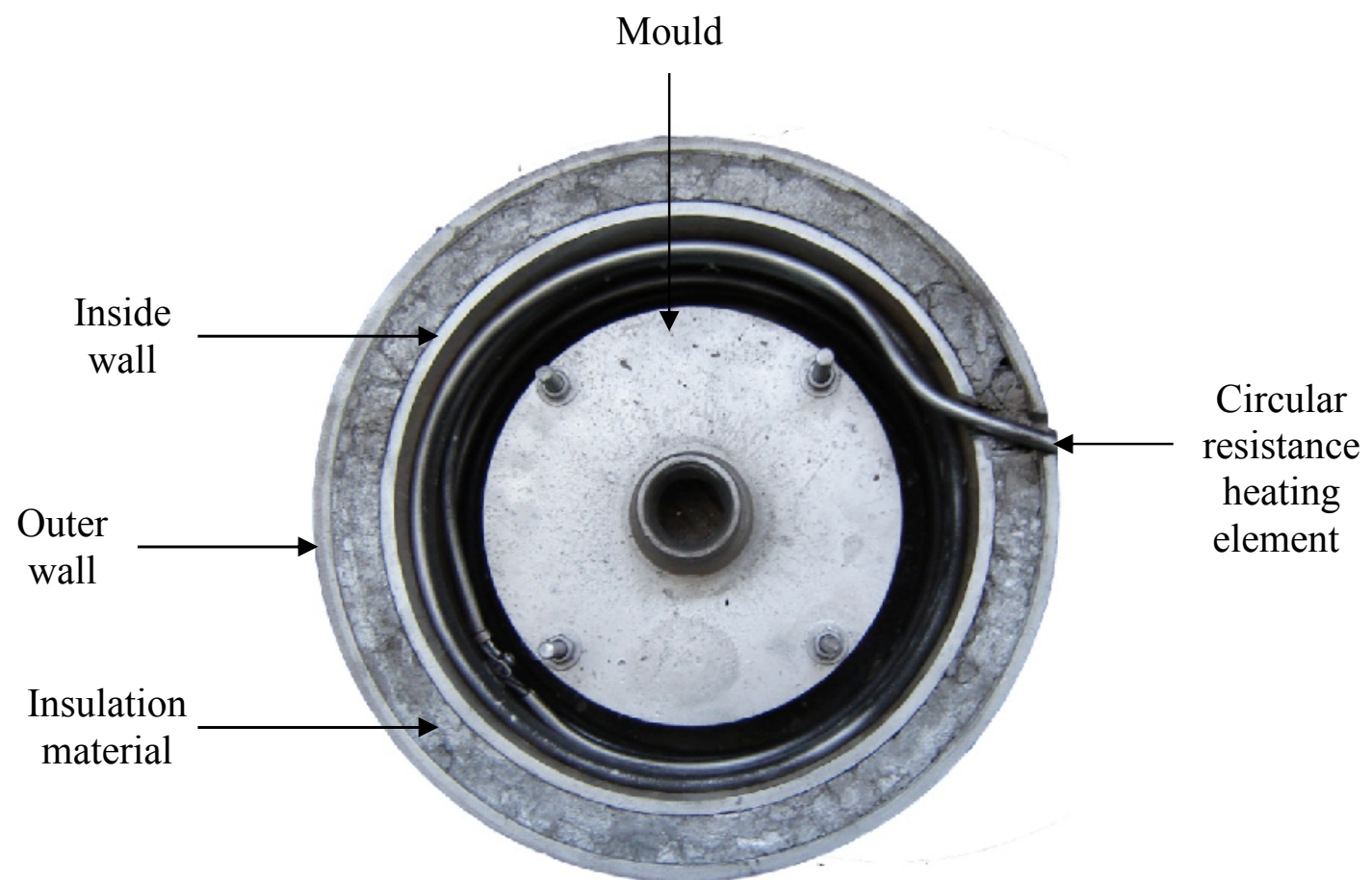

Figure (3): Photograph of heating unit of centrifugal casting set-up.

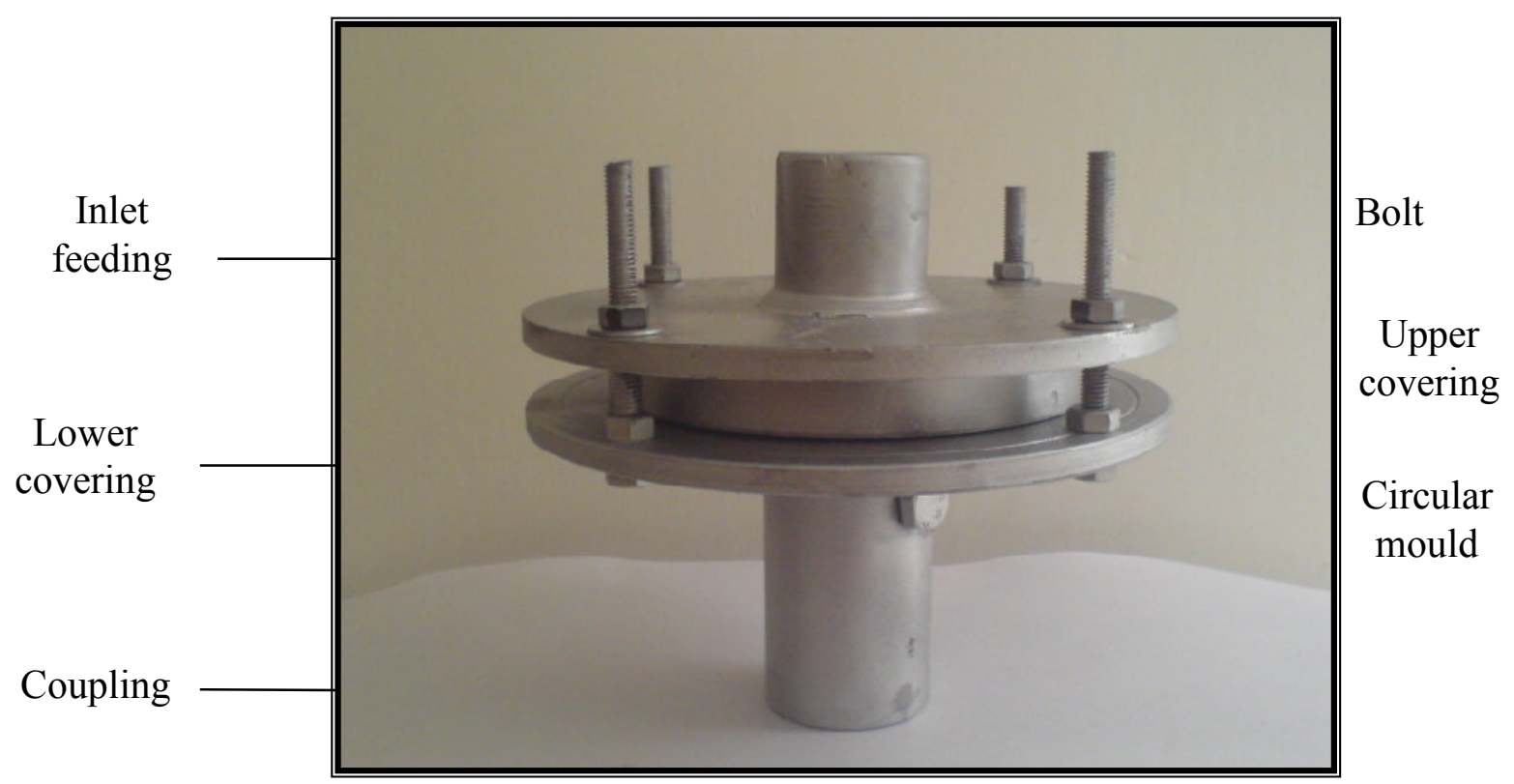

Figure (4): Photograph of the assembly of mould. 
Near about $1 \mathrm{~kg}$ of commercially aluminium alloy was melted in the clay-graphite crucible inside the main furnace at desired processing temperature of $850{ }^{\circ} \mathrm{C}$. Before any addition, the surface of the melt was cleaned well by skimming. At the same time, the mould of centrifugal casting was prepared and heated up to about $500{ }^{\circ} \mathrm{C}$. Prior to addition, the weighed amounts of $\mathrm{SiC}$ particles and magnesium were preheated to about $200{ }^{\circ} \mathrm{C}$ by putting them near the gate of the main furnace head. Magnesium was wrapped by aluminium foil to prevent it from oxidation and burning and added to the melt alloy at desired processing temperature. The preheated stirrer was used to mix and dispersed the magnesium in the molten alloy. After that, a desired amount of preheated SiC particles was added slowly at the rate of approximately $4 \mathrm{~g} / \mathrm{min}$ to the melt. The speed of stirrer was kept constant in all experiments at about $900 \mathrm{rpm}$. A digital strobometer was used to measure the stirring speed. The position of the stirrer inside the melt was always kept constant at the given level. The temperature of the melt was kept constant for all experiments and measured by using a temperature indicator connected to a chromel-alumel thermocouples placed at depth of 15-20 $\mathrm{mm}$ inside the melt during the stirring. The temperature of the melt slurry was maintained within $750{ }^{\circ} \mathrm{C}$ of the processing temperature. When the desired time (about 15-20 minute) elapsed, the speed of the stirrer was brought down slowly till stopped. Then, the clay-graphite crucible was removed from the main furnace by using a special designed hand lifting device. The melt slurry was, then, poured into a preheated centrifugal mould; the heaters are switch off. The centrifugal casting mould is rotated about 15 minute to ensure the solidification the cast FGCs. The pouring process was kept as close as possible to the top of the mould leaving only the necessary clearance to ensure minimum exposure of the slurry to the surrounding environment. No degassing practice of the slurry or melt was carried out at any stage of processing. In order to study the influence of the processing speed of mould on the microstructure and consequently on the mechanical properties, different speeds of rotation of the mould were used, namely 1000, 1250, 1500, 1750 and $2000 \mathrm{rpm}$.

Cast unreinforced alloys having chemical composition of aluminium, magnesium and silicon, similar to that of the matrix alloy of the cast FGCs, were prepared using the same setup and procedures stated above, but without any addition of SiC particles. These casting alloys were used for the comparison of the microstructures and the mechanical properties with those obtained in the different cast FGCS. Also, cast gravity composites having chemical composition and constituents nearly similar to that of the FGCs were prepared using the same set-up and procedures stated above, with the same amount of the $\mathrm{SiC}$ particles addition, but without rotation of the mould. Also, these casting composites were used for the comparison of the microstructures with those obtained in different cast FGCs and unreinforced alloys.

\section{4: Results and Discussion:}

Figures 5 and 6 show the dimensions of test specimen after machining out from the cast ingot and the scheme of sectioning the cast ingot of FGCs, gravity composite and unreinforced alloys was used to prepare specimens for metallographic studies respectively. The typical unetched optical microstructure of unreinforced alloy (AA4043) is shown in Fig.7. The microstructure consists of precipitates of intermetallic phase (Al-Si) forming due to the presence of silicon in the matrix alloy beyond the limit of solid solubility. Some dark areas of porosity are also visible. The cast unreinforced alloy has been prepared for comparison with the matrix microstructure of different cast FGCs. 
The typical unetached optical microstructure of cast particulate ordinary aluminium alloy based composite contained 33 vol.\% of reinforcing SiC particles and similarly processed as that of FGCs but without rotation of mould is shown in Fig.8. It is generally observed that the matrix alloy was enriched by the reinforcing SiC particles. Also, it is observed that the $\mathrm{SiC}$ particles are more or less uniformly distributed in the matrix alloy of cast composite. Some dark areas of porosity are also visible. The cast composite has been prepared for comparison with the different cast FGCs.

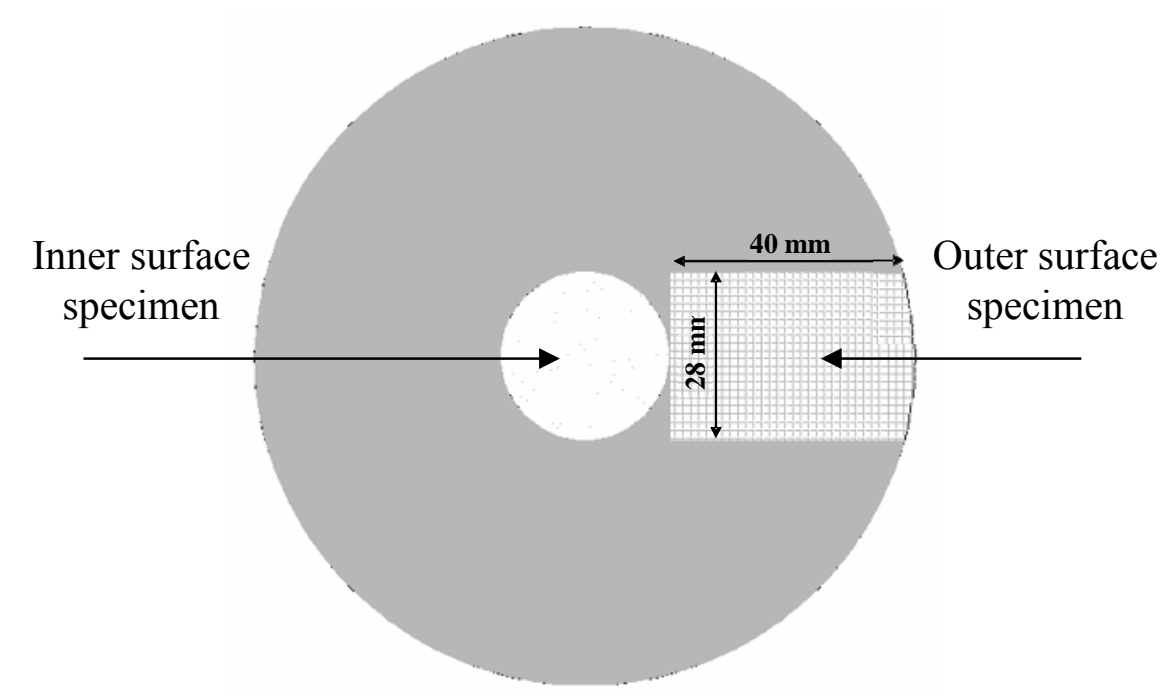

Figure (5): The test specimen after machining-out.

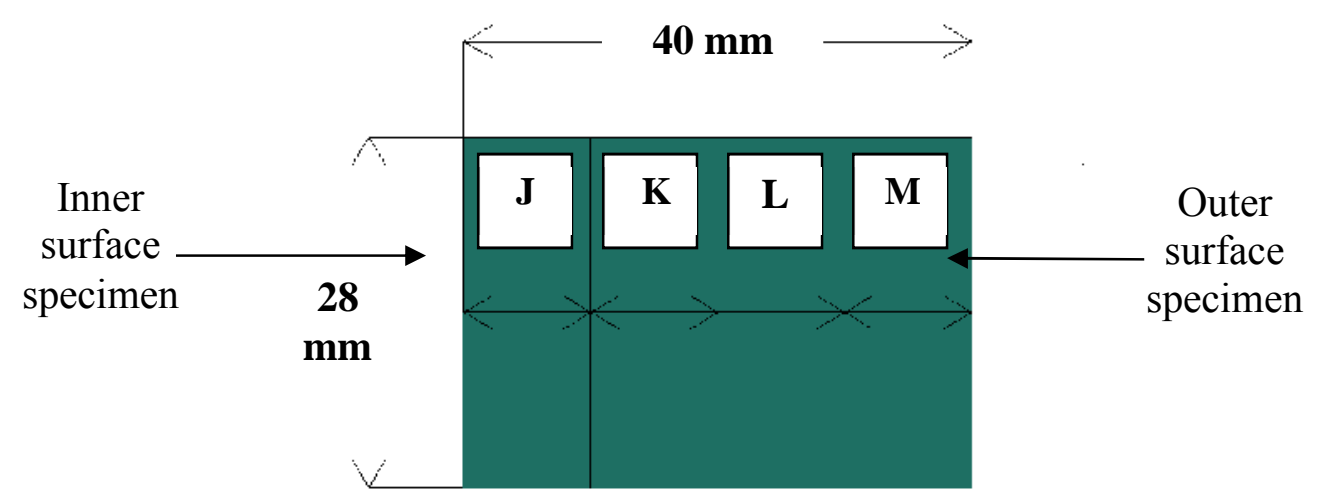

Figure (6): The specimen used in microstructure test, each section having an equal dimension of $10 \mathrm{~mm}$. 


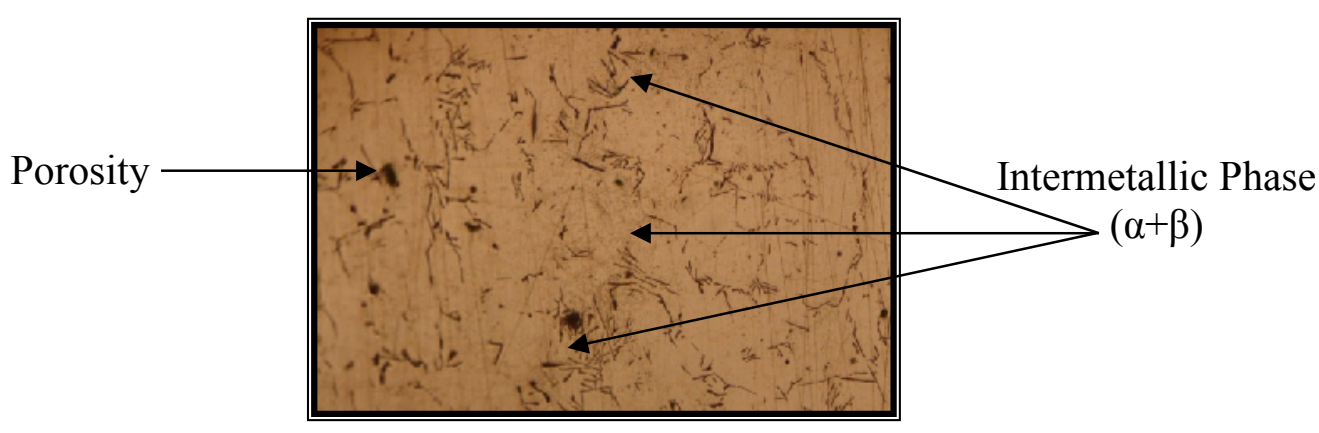

Figure (7): Typical unetched optical microstructure of unreinforced alloy, X85.

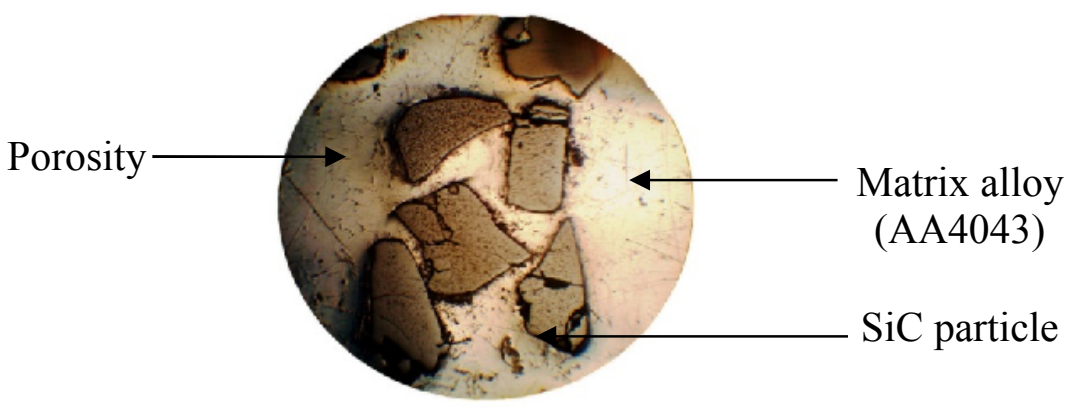

Figure (8): Typical unetched optical microstructure of cast partıculate composite containing $33 \mathrm{vol} \% \mathrm{SiC}$ particles without rotation of mould, X50.

Figure 9 shows a typical unetached optical microstructure of cast particulate aluminium alloy-based FGCs and it mainly consists of $\mathrm{SiC}$ particles imbedded in the matrix alloy. The interesting feature is the porosity attached to the reinforcing particles as shown in Fig.9. These porosities may use the surfaces of the poorly wetting particles as potent sites for bubble nucleation and the bubble may remain attached to these particles [10].

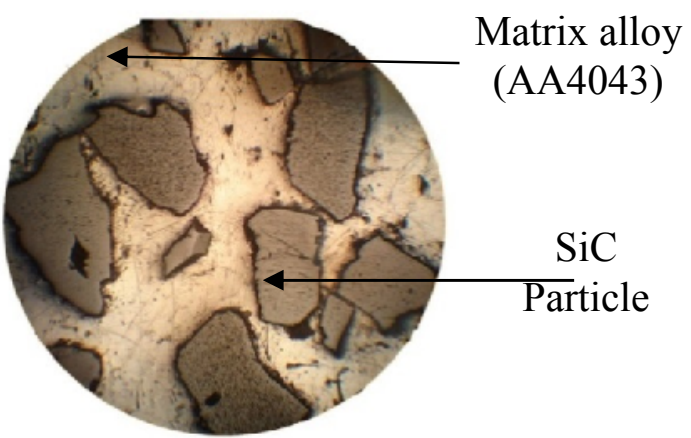

Figure (9): Typical unetched optical microstructure of cast FGCs containing 33 vol\% SiC particles, X50. reinforcing $\mathrm{SiC}$ particles, have been developed at different processing speeds of mould while the other processing parameters are maintained constants. The unetched optical particles distribution of FGCs cast at different processing speeds of mould are shown in Figs. 10-14. The particles distribution reported in the present study relate to those observed in the middle height segment of the cast specimen unless stated otherwise. It was observed that as the processing speed of mould increases, the amount of reinforcing particles in the outer region of the cast ingot increases. It is well known, that the moving direction of the reinforcing particle under the effect of centrifugal force is determined by the magnitude of the densities difference 


\section{Hamid: A preparation and Characterization of Functionally Graded Aluminum Alloy}

of particle and matrix alloy. Since, the density $\mathrm{SiC}$ is larger than that of the matrix alloy, more amount of $\mathrm{SiC}$ particles is expected that in the outer region obtained in high processing speed of mould as shown in Figs. 10-14.

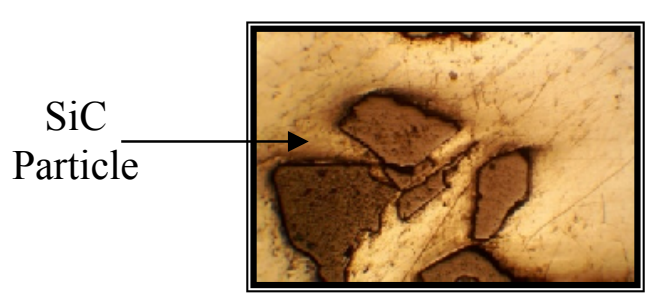

M- section

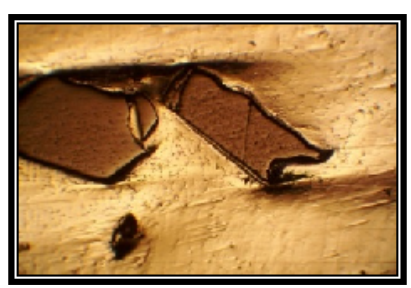

K- section

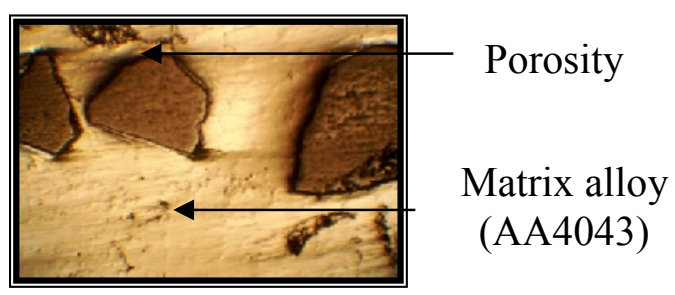

J- section

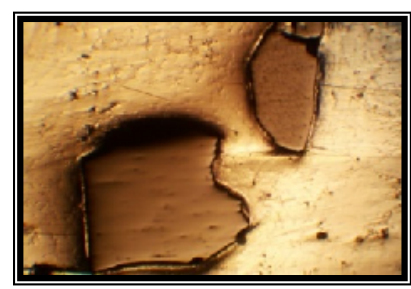

L- section

Figure (10): Unetched optical microstructure of cast FGCs containing

33 vol.\% of SiC particles, synthesized at processing speed of $1000 \mathrm{rpm}, \mathrm{X} 63$.

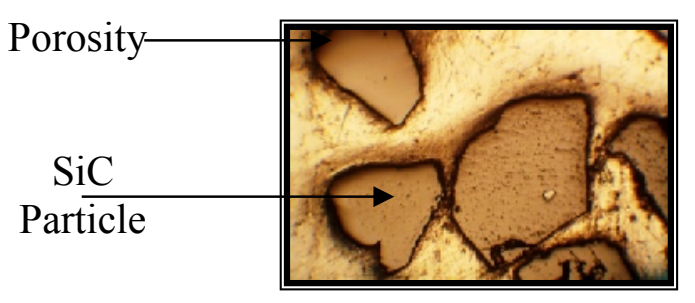

M- section

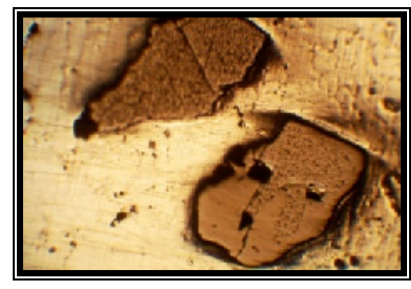

K- section

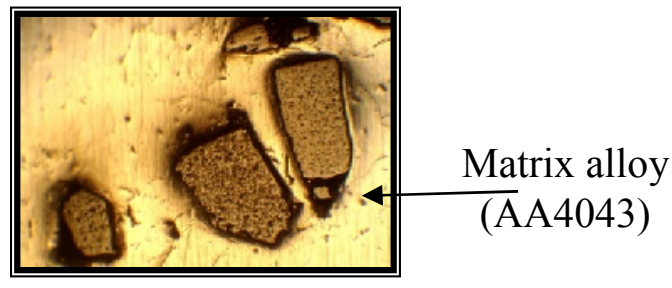

L- section

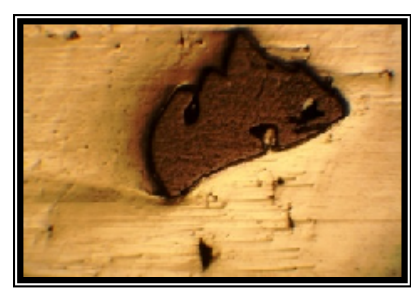

J- section

Figure (11): Unetched optical microstructure of cast FGCs containing $33 \mathrm{vol} . \%$ of $\mathrm{SiC}$ particles, synthesized at processing speed of $1250 \mathrm{rpm}, \mathrm{X} 63$. 


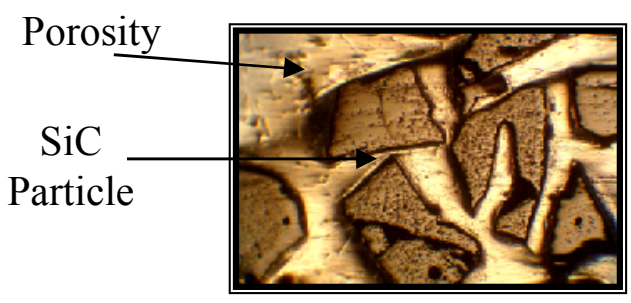

$\mathrm{M}$ - section

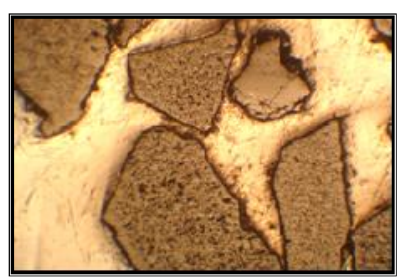

K- section

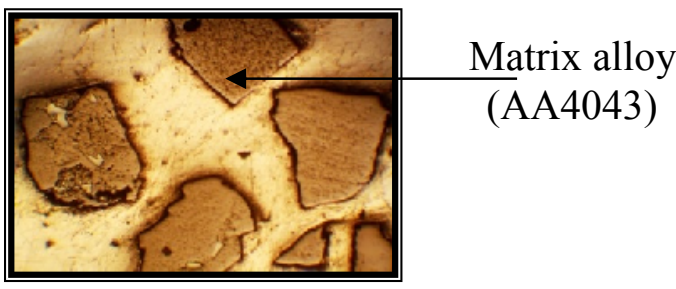

L - section

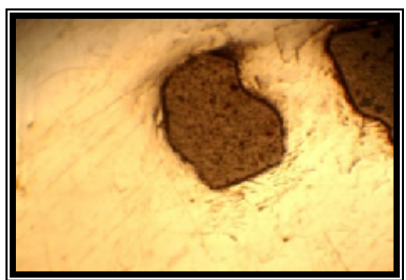

J- section

Figure (12): Unetched optical microstructure of cast FGCs containing 33 vol.\% of SiC particles, synthesized at processing speed of1500 rpm, X63.

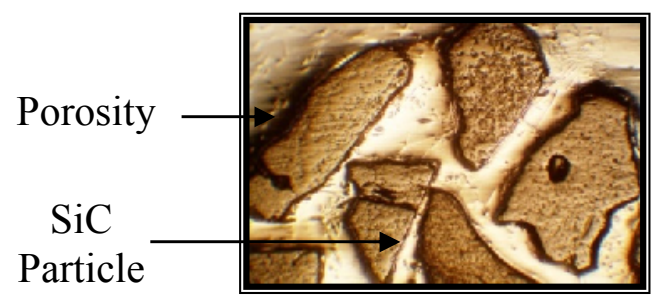

M- section

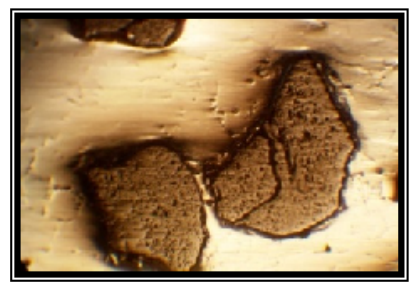

K- section

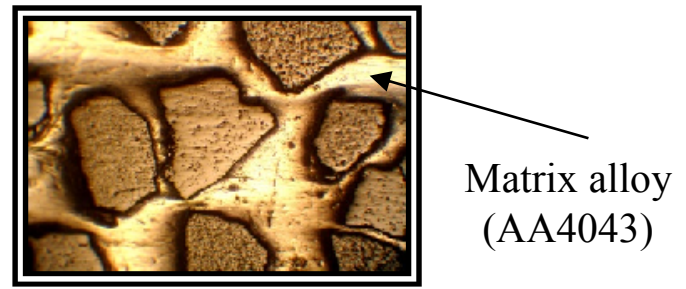

L- section

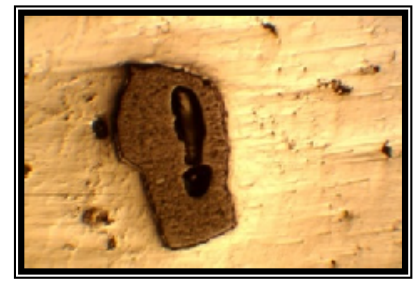

J- section

Figure (13): Unetched optical microstructure of cast FGCs containing $33 \mathrm{vol} . \%$ of $\mathrm{SiC}$ particles, synthesized at processing speed of1750 rpm, X63. 


\section{Hamid: A preparation and Characterization of Functionally Graded Aluminum Alloy}

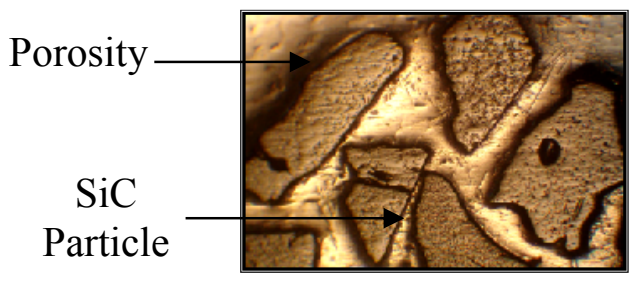

M- section

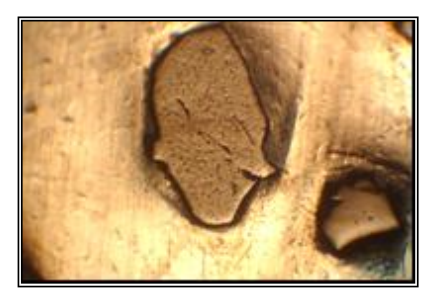

K- section

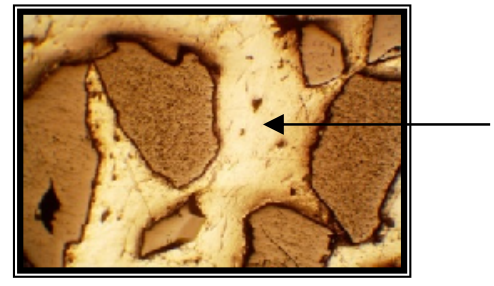

L- section

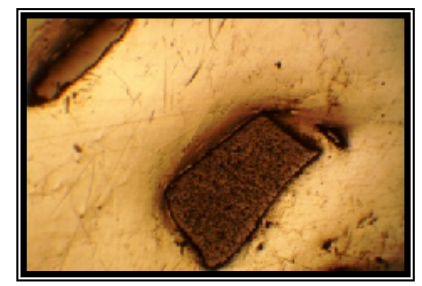

J- section
Matrix alloy

(AA4043)

Figure (14): Unetched optical microstructure of cast FGCs containing $33 \mathrm{vol} . \%$ of $\mathrm{SiC}$ particles, synthesized at processing speed of $2000 \mathrm{rpm}, \mathrm{X} 63$.

For comparison, cast alumimium alloy based composites have been developed and processed in a way similar to that of FGCs but without rotation of mould. Figure 15 shows the unetached optical particles distribution of cast Al-SiC composite. It is observed that the $\mathrm{SiC}$ reinforcing particle is more or less uniformly distributed from inner to outer region of the cast ingot.

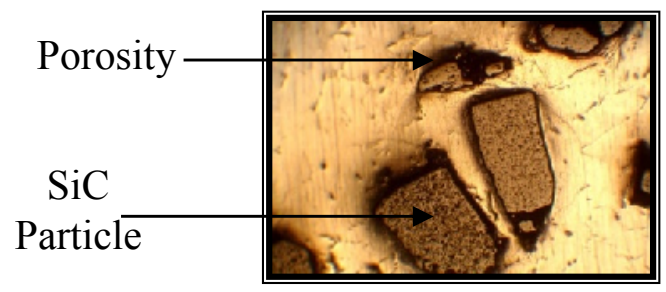

M- section

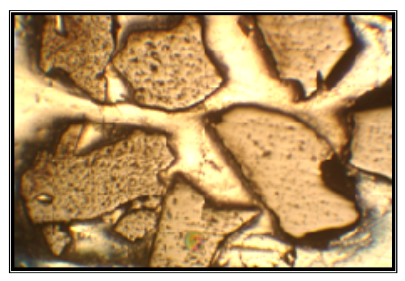

K- section

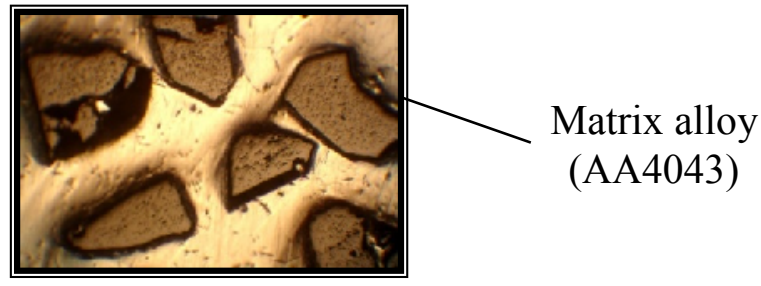

L- section

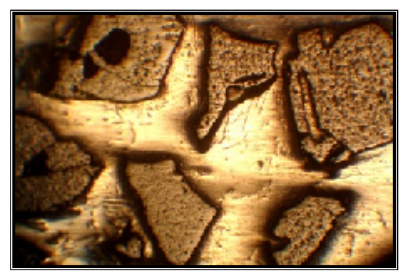

J- section

Figure (15): Unetched optical microstructure of cast (AA4043- SiC) composite without mould rotation at different regions from outer to inner region, $\mathrm{X} 63$. 
In the present study, the reinforcing particles phase was observed as grayish areas under optical microscope. Dark black areas were also observed and treated as porosity.

The image analyzer software program was used for estimating Area Fraction (Af) at each section for all FGCs and cast composite. Representative image had been selected at a given section in the cast FGCs specimen and the value of the analysis of these images was reported as the A $f$. Figure 16 shows the variation of A $f$ with increasing distance for different cast FGCs. It is observed from Fig. 16 that, for a given processing speed, the estimated Af increases significantly with increasing radial distance from inner to outer region for all different cast FGCs. Also, it is observed that the A $f$ increases significantly at outer region with increasing processing speed of mould from 1000 to $2000 \mathrm{rpm}$. At the same time, the Af decreases slightly at the inner region with increasing processing speed. Thus, the increased A $f$ at the outer region and reduced $\mathrm{A} f$ at the inner region, could be attributed to the movement of reinforcing particles. As the processing speed of mould increases, the effect of centrifugal force increases and the relatively densier $\mathrm{SiC}$ particles move outward and segregate at the outer region.

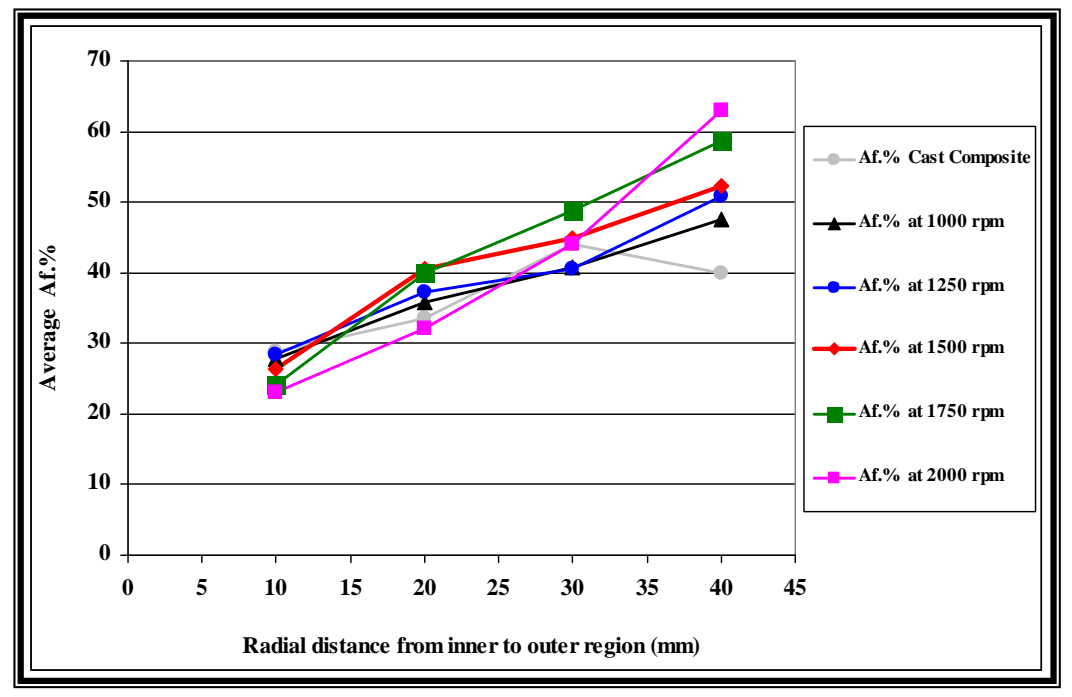

Figure (16): Area fractions (A $f$ ) for the all FGCs specimens at different processing speed of rotation mould and cast composite.

In order to identify the role of processing speed of mould on the A $f$, The A $f$ of the present cast FGCs has been compared with that observed in cast composite processed similarly as the cast FGCs but without any rotational speed of mould. The result of Af for cast composite is shown in the same figure. It is observed that the $\mathrm{A} f$ is more or less constant with increasing radial distance from inner to outer region. This indicates that there is more homogeneity in the distribution of reinforcing particles in the cast composite compared with that in FGCs.

The variation in Rockwell hardness with radial distance from the inner to the outer region for cast unreinforced alloy, cast composite and cast FGCs processed at 10001250 , 1500, 1750 and $2000 \mathrm{rpm}$, is shown in Fig. 17 (a and b); (a) for top surface and (b) for bottom surface. It is observed from the figure that hardness remains nearly constant with 
increasing radial distance from inner to the outer region for cast unreinforced alloy. Also, it is observed that there is little difference in hardness between top and bottom surfaces.

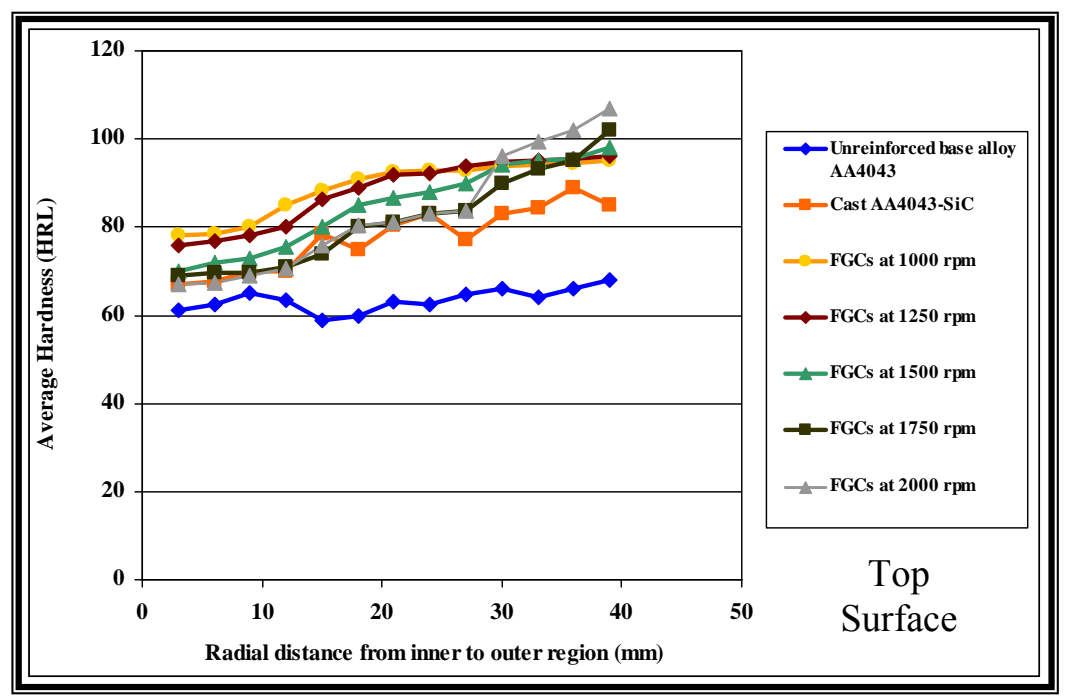

Figure (17a): The variation of average Rockwell (C) hardness with radial distance for cast unreinforced alloy, cast composite and all cast FGCs.

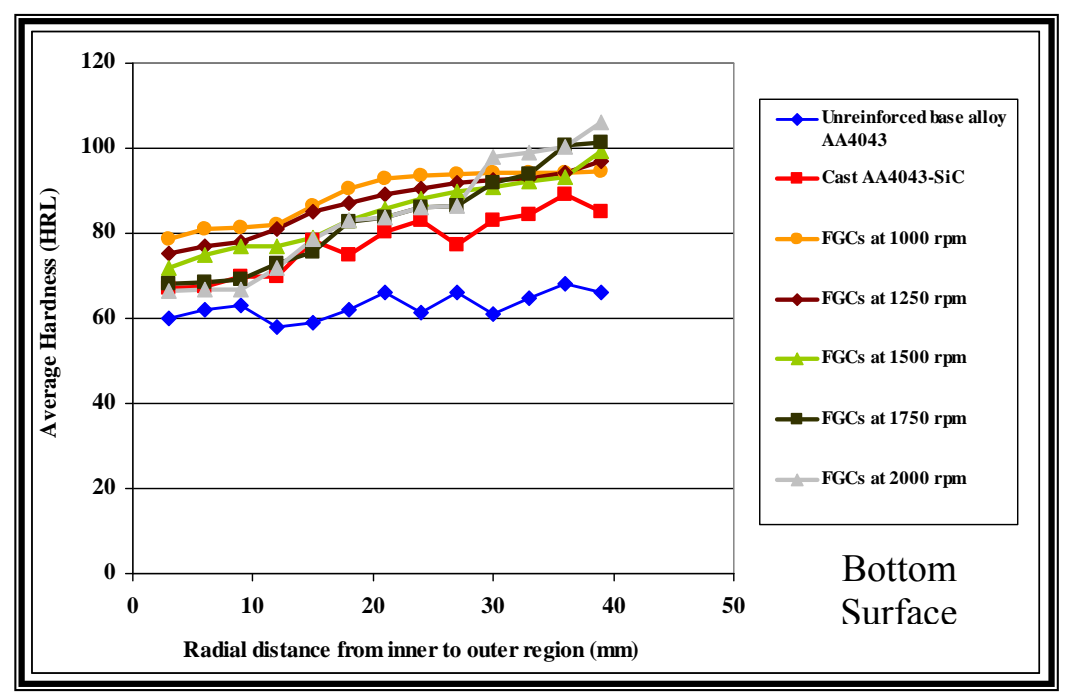

Figure (17b): The variation of average Rockwell (C) hardness with radial distance for cast unreinforced alloy, cast composite and all cast FGCs.

The variation in hardness with radial distance for cast composites similarly processed as that for unreinforced alloy and FGCs is also shown in the same figure. The hardness increases slightly with increasing radial distance. There is no significant difference in the average hardness between the top and bottom surfaces with increasing radial distance, as observed from Fig. 17. 
If one compares the hardness of cast unreinforced base alloy with that of cast composite containing 33 vol.\% reinforcing particles, it appears that the hardness of the cast composite increases significantly than that of cast unreinforced alloy and this could be attributed to the addition of the reinforcing particles in the matrix alloy.

For all cast FGCs, the hardness increases significantly with increasing radial distance for the top and the bottom surfaces of the cast specimen. At a given processing speed (say, for example, $2000 \mathrm{rpm}$ ), the hardness reached a maximum value at the outer region of cast FGCs and could be attributed to the segregation of the reinforcing particles at the outer region, the gradient in the value of hardness from inner to outer region is acute. The segregation of reinforcing particles accures mainly due to the effect of centrifugal force generated and applied to the semisolid mixture of slurry composite melt which lead to the formation of the desired composition gradient.

The observation of porosity content from the survey of images of different FGCs and composite shows that there is no significant difference in the porosity content, in both materials. But, in case of cast FGCs, the porosity, which is mainly originated from the gases dissolved in the melt during processing increases significantly with increasing distance from inner to outer region (as particle content increasing) of the resulting cast specimen. Also, it is observed that there is little increase in porosity content with increasing processing speed of mould, particularly at higher speed. Thus, the increased porosity content in the resulting cast FGCs, developed at higher processing speed of mould could be attributed to the increase in particle content at the outer region of the resulting cast specimen.

The hardness of cast FGCs are expected to be better than those of cast unreinforced alloy due to the reinforcement of matrix alloy by SiC particles. Also, it is expected that the mechanical properties to be better than those of cast composites for the same volume fraction of the reinforcing particles. The reason for this improvement is mainly due to less porosity content in FGCs as compared to that for cast composites.

\section{5- Conclusions:}

The main conclusions may be drawn from the current study are:

1- There is a considerable increases the hardness of cast composite over those of the cast unreinforced base alloy. This has been attributed to the reinforcement of matrix alloy by $\mathrm{SiC}$ particles.

2- Increasing processing speed of mould increases the extent of inhomogenity of the particle content along the radial distance of the cast FGCs.

3- Increasing processing speed of mould increases the hardness at the outer region of FGCs and reached maximum value at processing speed of $2000 \mathrm{rpm}$.

4- Increasing processing speed of mould decreases the hardness at the inner region of FGCs and reached minimum value at processing speed $2000 \mathrm{rpm}$.

5- Little differences in hardness from inner to the outer region of cast composite are possibly due to the inhomogeneous distribution of reinforcing particles and, also, possibly due to the dominating influence of porosity.

6- There are little differences in particle content between the top and bottom surfaces of all different cast FGCs processed at different processing speeds. 


\section{6- References:}

[1]- Donald, R. A., "The Science and Engineering of Materials" Third S.I Edition, United Kingdom (UK), p.549, (1998).

[2]- ASM Handbook committee "Composites" Vol. 21, p.130, (2001).

[3]- Gomes J.R., Ribeiro A.R, Vieira A.C, Miranda A.S and Rocha L.A, "Wear Mechanisms in Functionally Graded Aluminium Matrix Composites: Effect of the Lubrication by an Aqueous Solution" FCT- Portugal, pp.1-6, (2002).

[4]- James, P. S., Ashok, S, Stephan. D. A, Thomas.H. S and Steven. B. W "The Science and Design of Engineering Materials", $2^{\text {th }}$ Edition, United States, p.578, (1999).

[5]- Manoj Kumar B.V, Bikramjit .B, Murthy V.S.R and Manoj Gupta "The role of tribochemistry on fretting wear of $\mathrm{Mg}-\mathrm{SiC}$ particulate composites" Composites: Part (A) 36, pp. 13-23, (2005).

[6]- Zhang J, Wang Y. Q, Zhou B.L and Xing Q.W "Functionally graded Al/Mg $\mathrm{Mg}_{2} \mathrm{Si}$ in-situ composites, prepared by centrifugal casting" Journal of Materials Science, Vol.17, pp.16771679, (1998).

[7]- Yoshimi W, Ick S.K and Yasuyoshi F. "Microstructures of Functionally Graded Materials Fabricated by Centrifugal Solid-Particle and In-Situ Methods" Metals and materials International, Vol. 11, pp.391-399, (2005).

[8]- Zenon H.M.P "Fabrication and Characterization of functionally graded $\mathrm{Al} / \mathrm{AlB}_{2}$ matrix composites for high aerospace application using centrifugal casting" MSc.Thesis, Mechanical Engineering, University of Puerto Rico, pp.77-85, (2006).

[9]- Mohammed M.A.A "Study the Characterizations of Aluminium Alloy Based Functionally Graded Composites Produced by casting technique" MSc.Thesis, Mechanical Engineering, University of Mosul, pp.57-79, (2011).

[10]- Ray, S., "Cast metal matrix composites- Challenges in processing and design", Bulletin of Mater. Sci, Vol.18, No. 6, pp.693-707, (1995).

The work was carried out at the college of Engineering. University of Mosul 